\title{
Patterns of the ground states in the presence of random interactions: nucleon systems
}

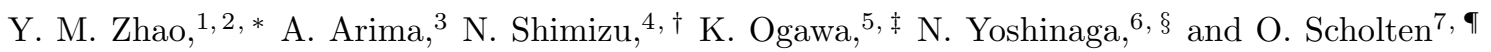 \\ ${ }^{1}$ Cyclotron Center, Institute of Physical Chemical Research (RIKEN), \\ Hirosawa 2-1, Wako-shi, Saitama 351-0198, Japan \\ ${ }^{2}$ Department of physics, Southeast University, Nanjing 210018 China \\ ${ }^{3}$ The House of Councilors, 2-1-1 Nagatacho, Chiyodaku, Tokyo 100-8962, Japan \\ ${ }^{4}$ Department of Physics, University of Tokyo, Bunkyo-ku, Tokyo 113-0033, Japan \\ ${ }^{5}$ Department of physics, Chiba university, Yayoi-cho 1-33, Inage, Chiba 263-8522, Japan \\ ${ }^{6}$ Department of Physics, Saitama University, Saitama 338 Japan \\ ${ }^{7}$ Kernfysisch Versneller Instituut, University of Groningen, 9747 AA Groningen, The Netherlands
}

(Dated: today)

\begin{abstract}
We present our results on properties of ground states for nucleonic systems in the presence of random two-body interactions. In particular we present probability distributions for parity, seniority, spectroscopic (i.e., in the laboratory framework) quadrupole moments and $\alpha$ clustering in the ground states. We find that the probability distribution for the parity of the ground states obtained by a two-body random ensemble simulates that of realistic nuclei with $A \geq 70$ : positive parity is dominant in the ground states of even-even nuclei while for odd-odd nuclei and odd-mass nuclei we obtain with almost equal probability ground states with positive and negative parity. In addition we find that for the ground states, assuming pure random interactions, low seniority is not favored, no dominance of positive values of spectroscopic quadrupole deformation is observed, and there is no sign of $\alpha$-clustering correlation, all in sharp contrast to realistic nuclei. Considering a mixture of a random and a realistic interaction, we observe a second order phase transition for the $\alpha$-clustering correlation probability.
\end{abstract}

PACS numbers: 21.60.Ev, 21.60.Fw, 05.30.Jp, 24.60.Lz

Keywords: Random Interactions; shell model; ground state deformation; $\alpha$-clustering correlation

\section{INTRODUCTION}

It was discovered in Ref. [1] that the dominance of spin zero ground states ( 0 g.s. ) can be obtained by diagonalizing a scalar two-body Hamiltonian with random valued matrix elements, a so-called two-body random ensemble (TBRE) Hamiltonian. The 0 g.s. dominance was soon confirmed in Ref. [2] for sd-boson systems. These feature was found to be robust and insensitive to the detailed statistical properties of the random Hamiltonian, suggesting that the 0 g.s. dominance arises from a very large ensemble of two-body interactions other than a simple monopole paring interaction. An understanding of this discovery is very important, because this observation seems to be contrary to what is traditionally assumed in nuclear physics, where the 0 g.s. dominance in even-even nuclei is usually explained as a reflection of attractive pairing interaction between like nucleons.

There are a lot of efforts to understand this observation but a fundamental understanding is still out of reach [3]. There are also many works [4] studying other robust phenomena of many-body systems in the presence of the TBRE. For example, the studies of odd-even staggering

\footnotetext{
*Electronic address: ymzhao@riken.jp

†Electronic address: shimizu@nt.phys.s.u-toyo.ac.jp

‡Electronic address: ogawa@physics.s.chiba-u.ac.jp

$\S$ Electronic address: yosinaga@phy.saitama-u.ac.jp

ฯElectronic address: scholten@kvi.nl
}

of binding energies, generic collectivity, the behavior of energy centroid of fixed spin states, correlation, etc.

The purpose of the present paper is to focus our attention on some physical quantities for the ground states which have not been studied, specifically, parity, seniority, spectroscopic quadrupole moments (i.e., measured in the laboratory framework), and $\alpha$-clustering probability. For realistic nuclei these quantities show a very regular pattern. In this paper we shall discuss whether these regular patterns are robust in the presence of random interactions.

As well known, all even-even nuclei have positive parity ground states (i.e., 100\%) whereas the ground states of nuclei with odd mass numbers have only a slightly higher probability for positive parity than for negative parity. Odd-odd nuclei have almost equal probabilities for positive and negative-parity ground states $(\sim 50 \%)$. The statistics for the ground state parity of nuclei with mass number $A \geq 70$ are summarized in Table I As the first subject we will study the ground-state parity distribution using random interactions.

The next subject that we shall discuss in this paper is the distribution of seniority in the ground states. Seniority [8] has been proven a very relevant concept in nuclear physics, in particular for spherical or transitional nuclei. Seniority $(v)$ is uniquely defined for a single- $j$ shell; it was generalized to the case of many- $j$ shells by Talmi in Ref. [9]. In Refs. 1, 10] it was reported that the pairing phenomenon seems favored simply as a consequence of the two-body nature of the interaction. The "pairing" of Refs. 1, 10] was defined as a large matrix element of the 
TABLE I: The positive parity distribution of the ground states of atomic nuclei. We included all ground state parities of nuclei with mass number $A \geq 70$. The data are taken from Ref. [6]. We haven't taken into account those nuclei for which the ground state parity was not measured.

\begin{tabular}{cccc}
\hline \hline counts & even-even & odd- $A$ & odd-odd \\
\hline verified (+) & 487 & 281 & 118 \\
verified (-) & 0 & 215 & 104 \\
\hline tentative (+) & 0 & 159 & 70 \\
tentative (-) & 0 & 126 & 60 \\
\hline \hline
\end{tabular}

$S$ pair annihilation operator between the ground states of a $n$ fermion system to a $n-2, n-4 \cdots$ systems, where the $S$ pair structure is determined using the procedure of Talmi's generalized seniority scheme. This indicated that the $S$-pair correlation is dominant for the spin- 0 g.s. of these systems. An examination of this "pairing" correlation of fermions in a single- $j$ shell in Ref. [5] showed that, however, an enhanced probability for low seniority in the spin- 0 g.s. is not observed in most of the calculations using a TBRE hamiltonian. For many- $j$ shells, there have been few discussions to clarify this point so far.

Another subject that we shall discuss is the $\alpha$ clustering correlation in the presence of random interactions. The importance of the $\alpha$-clustering correlation in light and medium nuclei have been emphasized by many authors [1]. The $\alpha$-clustering correlation also plays an important role in astrophysical processes such as the Salpeter process in the formation of ${ }^{12} \mathrm{C}$. Many calculations of the low-lying states, using the anti-symmetrized molecular dynamics model, have been done in recent years [12] to study the $\alpha$-clustering and other clustering correlations for both stable and unstable light nuclei. $\alpha$ cluster condensation was suggested by Horiuchi, Schuck and collaborators in Ref. [13]. As a function of the mixing of a realistic and the TBRE interaction a phase transition is observed in the $\alpha$-clustering probability in the ground state.

In this paper we also discuss the spectroscopic quadrupole moments (i.e., measured in the laboratory framework) of the ground states. A positive value for spectroscopic quadrupole deformation is dominant in the low-lying states of atomic nuclei. Recently it has been argued in Ref. 14 that this is due to the interference of spin-orbit and $l^{2}$ terms of the Nilsson potential.

Our calculations are based on the use of TBRE interactions. The single-particle matrix elements are set to be zero. The Hamiltonian that we use conserves the total angular momentum and isospin:

$$
\begin{aligned}
& H=\sum_{j_{1} j_{2} j_{3} j_{4}}^{J T} \sqrt{2 J+1} \sqrt{2 T+1} G_{j_{1} j_{2} j_{3} j_{4}}^{J T} \\
& \frac{1}{\sqrt{1+\delta_{j_{1} j_{2}}} \sqrt{1+\delta_{j_{3} j_{4}}}}
\end{aligned}
$$

$$
\left(\left(a_{j_{1} t}^{\dagger} \times a_{j_{2} t}^{\dagger}\right)^{(J T)} \times\left(\tilde{a}_{j_{3} t} \times \tilde{a}_{j_{4} t}\right)^{(J T)}\right)^{(00)}
$$

where these $G_{j_{1} j_{2} j_{3} j_{4}}^{J T}$ are defined by $\left\langle j_{1} j_{2} J T|V| j_{3} j_{4} J T\right\rangle$ and follow the following distribution,

$$
\rho\left(G_{j_{1} j_{2} j_{3} j_{4}}^{J T}\right)=\frac{1}{\sqrt{2 \pi x}} \exp \left(-\frac{\left(G_{j_{1} j_{2} j_{3} j_{4}}^{J T}\right)^{2}}{2 x}\right)
$$

with

$$
x=\left\{\begin{array}{ll}
1 & \text { if }\left|\left(j_{1} j_{2}\right) J T\right\rangle=\left|\left(j_{3} j_{4}\right) J T\right\rangle \\
\frac{1}{2} & \text { otherwise }
\end{array} .\right.
$$

The Hamiltonian such defined is called a TBRE Hamiltonian. Here $j_{1}, j_{2}, j_{3}$ and $j_{4}$ denote the respective singleparticle orbit, $J(T)$ denotes the total angular momentum (isospin) of two nucleons. For each system 1000 runs of calculations are performed in order to accumulate stable statistics. $N_{p}$ and $N_{n}$ refer to the number of valence protons and neutrons, respectively.

This paper is organized as follows. In Section II we present our results for parity distributions for a variety of systems. In Section [II we discuss the distribution of seniority in the ground states using random interactions. In Section $\square$ we show our results for spectroscopic quadrupole moment in the ground states which suggests prolate or oblate shapes. In Section $\nabla$ we discuss the $\alpha$ clustering correlation in the ground states. The summary will be given in Section DI.

\section{PARITY}

We select four model spaces for studying the parity distribution in the ground states obtained by random interactions:

A Both protons and neutrons are in the $f_{\frac{5}{2}} p_{\frac{1}{2}} g_{\frac{9}{2}}$ shell which corresponds to nuclei with both proton number $\mathrm{Z}$ and neutron number $\mathrm{N} \sim 40$;

B Protons in the $f_{\frac{5}{2}} p_{\frac{1}{2}} g_{\frac{9}{2}}$ shell and neutrons in the $g_{\frac{7}{2}} d_{\frac{5}{2}}$ shell which correspond to nuclei with $\mathrm{Z} \sim 40$ and $N \sim 50$;

C Both protons and neutrons are in the $h_{\frac{11}{2}} s_{\frac{1}{2}} d_{\frac{3}{2}}$ shell which correspond to nuclei with $\mathrm{Z}$ and $\mathrm{N} \sim 82$;

D Protons in the $g_{\frac{7}{2}} d_{\frac{5}{2}}$ shell and neutrons in the $h_{\frac{11}{2}} s_{\frac{1}{2}} d_{\frac{3}{2}}$ shell which correspond to nuclei with $\mathrm{Z} \sim$ 50 and $N \sim 82$.

These four model spaces do not correspond to a complete major shell but have been truncated in order to make the calculations feasible. These truncations are based on the sub-shell structures for the involved single-particle levels. We study the dependence on valence-proton number $N_{p}$ and valence-neutron number $N_{n}$ in these four model 
FIG. 1: Number of states with total angular momentum $I$ (denoted as $D(I)$ ) versus $I$. One sees that the $D(I)$ of positive parity levels and that of negative parity levels are very close to each other. (a) two protons in the $1 g_{9 / 2} 2 p_{1 / 2} 1 f_{5 / 2}$ shell and four neutrons in the $2 d_{5 / 2} 1 g_{7 / 2}$ shell; (b) two protons and two neutrons in the $1 g_{9 / 2} 2 p_{1 / 2} 1 f_{5 / 2}$ shell; (c) two protons and three neutrons in the $1 g_{9 / 2} 2 p_{1 / 2} 1 f_{5 / 2}$ shell; (d) three protons in the $1 h_{11 / 2} 3 s_{1 / 2} 2 d_{5 / 2}$ shell and three neutrons in the $2 d_{5 / 2} 1 g_{7 / 2}$ shell.

TABLE II: The positive parity probability for the ground states (in \%). In brackets the number of neutrons and protons $\left(N_{p}, N_{n}\right)$ is given for each configuration.

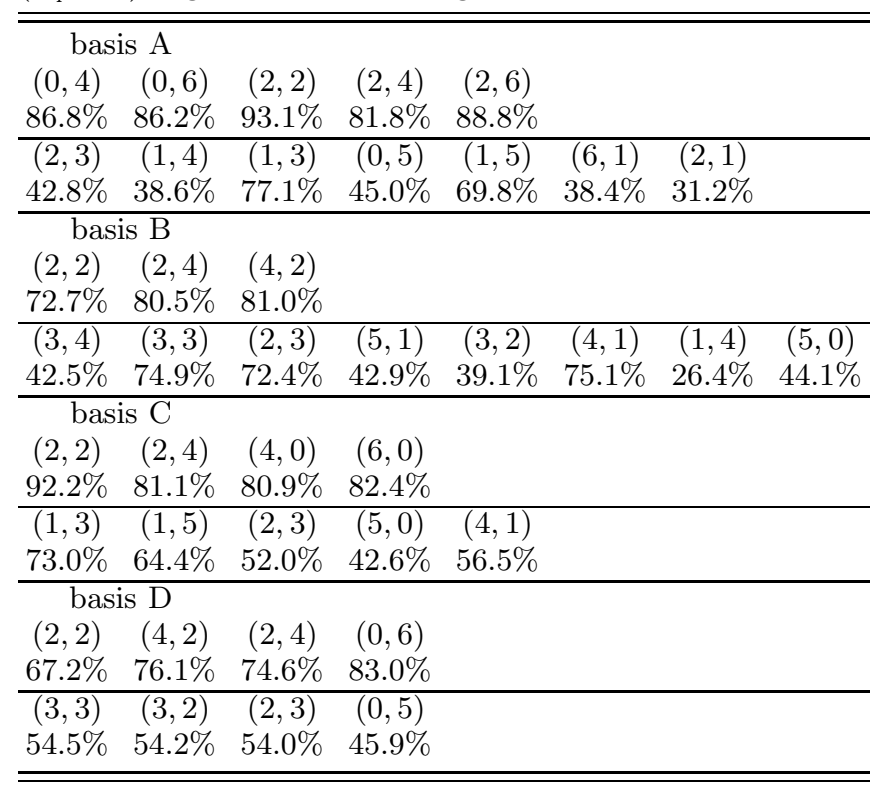

spaces. It is noted that the number of states (denoted as $D(I))$ for positive and negative parity are very close for all these examples. The $D(I)$ 's for a few examples are shown in Fig. (11). One thus expects that the probability of the ground states with positive parity is around $50 \%$, if one assumes that each state of the full shell model space is equally probable in the ground state.

The calculated statistics for the parity of the ground states, using a TBRE Hamiltonian, is given in Table This clearly shows that positive parity is favored, and dominant for most examples, for the ground states of even-even nuclei in the presence of random interactions.

The statistics for nuclei with odd mass numbers and those with odd values for both $N_{p}$ and $N_{n}$ is also given in Table III This statistics shows that the probabilities to have positive or negative parity in the ground states are almost equal with some exceptions. In general, there is no favoring for either positive parity or negative parity in the ground states of odd mass nuclei and doubly odd nuclei in the presence of random interactions. It is noted that these calculations are done for the beginning of the shell. For the end of the shell the results show a similar trend.

We also find that the above regularities for parity distributions also hold for very simple cases: single-closed two- $j$ shells with one positive-parity and one with negative parity. We have checked explicitly the cases for $\left(2 j_{1}, 2 j_{2}\right)=(9,7),(11,9),(13,9),(11,3),(13,5),(19,15)$, $(7,5),(15,1)$. The statistics is very similar to the above results: The probability of ground states with positive parity is about $85 \%$ for an even number of nucleons and about $50 \%$ for an odd number of nucleons.

It is interesting to note that for all even-even nuclei the $P\left(0^{+}\right)$is usually two orders in magnitude larger than $P\left(0^{-}\right)$. It would be very interesting to investigate the origin of the large difference for $P(0)$ for positive and negative parity states, i.e., why the $0^{-}$is not favored in the ground states. As is the case for an odd number of bosons with spin $l$ 7], spin $I=0$ is not a sufficient condition to be favored in the ground states of a manybody system in the presence of the random interactions. It should be noted that for the quantum numbers of a realistic g.s. not only $I=0$ is required but also positive parity.

One simple and schematic system to study the parity distribution of the ground states in the presence of random interactions is the $s p$-bosons system. First, we note that a $s p$-boson system with an odd value of $n$ has the same number of states with positive and negative parity; while for an even value of $n$ there are slightly more states with positive parity (the difference is only $n+1, n$ is the number of $s p$ bosons of the system). The calculated results of Ref. 15. showed that when the number $n$ of $s p$ bosons is even, the dominant $I$ in the ground states is 0 or $n$ (about 99\%), with positive parity (parity for $s p$ bosons is given by $\left.(-)^{I}\right)$ dominance. When the number of $n$ is odd, only about $50 \%$ of the ground states in the ensemble have spin- 0 , and about $50 \%$ have $I=1$ or $I=n$ ground states. This leads to about equal percentages for positive and negative parity ground states. This pattern is very similar to that observed for fermion systems.

\section{SENIORITY}

In this section, we discuss the distribution of the seniority, the number of particles not pairwise coupled to angular momentum 0 , of the ground states of nuclei in the 


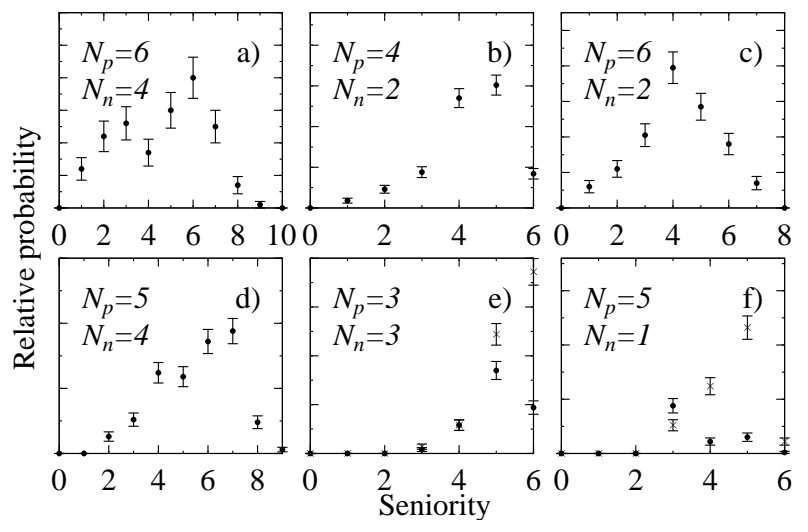

FIG. 2: Distribution of seniority in the ground states with spin zero for even-even nuclei (refer to panels (a), (b), and (c) ), spin $I=j_{1}, j_{2}, j_{3}$ for odd- $A$ case (refer to panel (d) ) or spin $I=1,0$ for odd-odd nuclei (refer to panels (e) and (f) ). The error bar is defined by the square root of the count (statistics) for each seniority bin (step width is 1 ). The dominance of seniority zero components of ground states is not observed.

$s d$ shell in the presence of random interactions. Because seniority is used in classifying the states in our basis, we define the expectation value for seniority in the ground states as follows 16.

$$
\langle v\rangle=\sum_{i} f_{i}^{2} v_{i}
$$

where $f_{i}$ is the amplitude of the $i$-th component in the ground state wave function, and $v_{i}$ is the seniority number of the corresponding component.

For even-even nuclei we consider the spin- 0 g.s. because previous discussions [5, 10] were focused on spin- 0 ground states. For odd-mass nuclei we consider the $I=1 / 2,3 / 2$, and $5 / 2$ ground states, because these spin $I$ 's are equal to the angular momenta of the single-particle levels in the $s d$ shell and are favored as the ground states in the presence of random interactions. For odd-odd nuclei in this section we consider the ground states with $I=1$ (most favored) and $I=0$ states. The examples that we have calculated include $\left(N_{p}, N_{n}\right)=(0,4),(0,6),(2,2)$, $(2,4),(2,6),(4,6),(0,5),(2,3),(2,5),(4,3),(4,5),(3,3)$, $(1,5),(3,5)$.

Typical examples of the distribution of average seniority $(\langle v\rangle)$ in the ground states are shown in Fig. (2) in arbitrary units (i.e. relative probability). The figure shows that for none of the cases a small value for $\langle v\rangle$ is preferred. These distributions of seniority in the ground states show that the large amplitude of for $S$-pair operator between the spin-0 g.s. of a $n$-nucleon system and that of a $(n+2)$ nucleon system, as observed in Ref. [10], should not be understood as an indication of large $S$ pair condensate in the spin- 0 g.s. of TBRE Hamiltonians. Further studies are necessary to understand the implications of Ref. [10].

\section{SPECTROSCOPIC QUADRUPOLE MOMENT}

In this section we study the quadrupole moments $Q$ of the ground or low-lying states. If the ground state spin $I$ is smaller than one (i.e., 0 or $1 / 2$ ), the external quadrupole-moment necessarily vanishes (even though there could be a finite intrinsic moment) because the triangle relationship of angular momentum coupling cannot be obeyed by the two $I$ 's $\left(I \leq \frac{1}{2}\right)$ and the angular momentum for quadrupole operator $(=2)$. For these cases one can use an alternative, namely, the quadrupole moment of the next lowest state with $I>\frac{1}{2}$. For all cases that we have checked, it is found that the essential statistics for positive and negative quadrupole moments obtained by this alternative is very close to those obtained by neglecting cases with ground state $I<1 / 2$. In this paper we show the statistics which do not includes the runs of spin -0 and spin $-1 / 2$ ground states. The total number of calculated external quadrupole moments is thus much less than 1000 . We note that a negative external quadrupole moment implies a positive quadrupole moment in the intrinsic frame and thereby a prolate deformation.

The external quadrupole moment is defined by

$$
Q=\left\langle\beta I\left|r^{2} Y_{2 M}\right| \beta I\right\rangle
$$

for both proton and neutron degrees of freedom. In Eq. (5) $|\beta I\rangle$ is the wave function of the ground state. In this paper "Q" will be used to refer to the external quadrupole moment following from Eq. (5).

We have calculated "Q" for a number of cases in the $s d$ shell and for several fillings of the four single-particle bases mentioned in Section III The results are given in Table III One sees that negative values for $Q$ (corresponding to prolate deformations) are dominant with two exceptions, $\left(N_{p}, N_{n}\right)=(4,3),(6,5)$ in the $s d$ shell. In general we observe that for the $s d$ shell the statistics for positive and negative values for $Q$ are comparable if $N_{p}$ and/or $N_{n}$ are close to their mid-shell values.

From Table III we conclude that at the beginning of the shell negative values for $Q$ are dominant, while at the end of the shell positive value dominate. This is similar to the result for a harmonic oscillator potential, for which prolate deformation occurs at the beginning and oblate deformation at the end of the shell [17].

\section{V. $\alpha$-CLUSTERING}

It was shown in Ref. [18] that the essential parts of the $I=0, T=0$ ground state for ${ }^{20} \mathrm{Ne}$ with two protons and two neutrons in the $s d$ shell is dominated by components with highest orbital symmetry, [4]; $91.8 \%$ of the ground state is given by components with orbital symmetry [4] which corresponds to a pure $\alpha$-clustering configuration. One may use the expectation value of the Majorana interaction, $P_{M}$, as the fingerprint for the $\alpha$-cluster wave 
TABLE III: The number of cases with positive (negative) external quadrupole moments are given in bold (italic) font, respectively. We omitted the cases for which the spin of the ground state is less than 1 , see the text for further details.

\begin{tabular}{|c|c|c|c|}
\hline \multicolumn{4}{|c|}{ both protons and neutrons in the $s d$ shell } \\
\hline$\left(N_{p}, N_{n}\right)$ & $(2,1)$ & $(2,3)$ & $(2,5)$ \\
\hline$\left(N_{p}, N_{n}\right)$ & $\begin{array}{c}280 \\
(2,1)\end{array}$ & $\begin{array}{c}338 \\
(4,3)\end{array}$ & $306 \quad 402$ \\
\hline$\left(N_{p}, N_{n}\right)$ & $\begin{array}{c}\mathbf{2 8 7} \\
(6,1)\end{array}$ & $\begin{array}{c}434 \\
(6,3)\end{array}$ & $\begin{array}{ll}320 & 370 \\
(6,5)\end{array}$ \\
\hline & 201 & 400 & 420 \\
\hline \multicolumn{4}{|c|}{ Basis (A); protons and neutrons in $f_{\frac{5}{2}} p_{\frac{1}{2}} g_{\frac{9}{2}}$} \\
\hline$\left(N_{p}, N_{n}\right)$ & $(1,2)$ & $(1,3)$ & $(1,4)$ \\
\hline$\left(N_{p}, N_{n}\right)$ & $\begin{array}{c}267 \\
(1,6)\end{array}$ & $\begin{array}{cc}283 & 481 \\
(2,3)\end{array}$ & $246 \quad(0,5)^{535}$ \\
\hline & $\mathbf{2 0 7} \quad 566$ & $\mathbf{2 8 4} \quad 564$ & 447 \\
\hline \multicolumn{4}{|c|}{ Basis (B); protons $\left(f_{\frac{5}{2}} p_{\frac{1}{2}} g_{\frac{9}{2}}\right)$, neutrons $\left(g_{\frac{7}{2}} d_{\frac{5}{2}}\right)$} \\
\hline$\left(N_{p}, N_{n}\right)$ & $(1,4)$ & $(4,1)$ & $(2,4)$ \\
\hline$\left(N_{p}, N_{n}\right)$ & $\begin{array}{c}\mathbf{3 7 4} \quad 507 \\
(3,4)\end{array}$ & $\begin{array}{c}\mathbf{2 7 8} \\
(4,3)\end{array}$ & $(6,1)^{428}$ \\
\hline & $\mathbf{2 7 8} 620$ & 330 & 233 \\
\hline \multicolumn{4}{|c|}{ Basis (C); protons and neutrons in $s_{\frac{1}{2}} d_{\frac{3}{2}} h_{\frac{11}{2}}$} \\
\hline$\left(N_{p}, N_{n}\right)$ & $(2,3)$ & $(2,5)$ & $(4,3)$ \\
\hline$\left(N_{p}, N_{n}\right)$ & $\begin{array}{c}\mathbf{2 3 1} \\
(5,1)\end{array}$ & $\begin{array}{cc}238 & 472 \\
(5,0) & \end{array}$ & $(3,3)^{498}$ \\
\hline & $\mathbf{2 1 3} 628$ & 212 & 349 \\
\hline \multicolumn{4}{|c|}{ 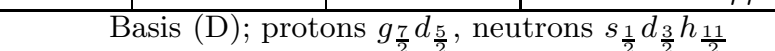 } \\
\hline$\left(N_{p}, N_{n}\right)$ & $(14,13)$ & $(15,12)$ & \\
\hline & 781 & $610 \quad 333$ & \\
\hline
\end{tabular}

function. Another similar example is the $I=0, T=0$ ground state for ${ }^{8} \mathrm{Be}$ with two protons and two neutrons in the $p$-shell. If one uses Cohen-Kurath interaction, one sees that the expectation value of $P_{M}$ is -5.76 , close to -6 which is the eigenvalue of Majorana force. The overlap between the g.s. wave function obtained by diagonalizing the Cohen-Kurath interaction for ${ }^{8} \mathrm{Be}$ and that for exact SU(4) symmetry (namely, full symmetry [4] for the ground state) is 0.97 . This dominance full symmetry [4] in the $I=0$ and $T=0$ ground state of these nuclei with respect to the permutation of orbital degree of freedom is an indication of $\alpha$-clustering correlation from the perspective of the shell model. In this paper we concentrate on these two examples using random interactions.

To set the scale, we can calculate the matrix element of $P_{M}$ in the $I=T=0$ (spin-isospin singlet) ground state by assuming that all the possible $I=T=0$ states with different symmetries with respect to exchanging the orbital degree of freedom appear with equal-probably. We call the $P_{M}$ such obtained the geometric $P_{M}$. To do so one needs the number of $(I=0, T=0)$ states for each symmetry of orbital degree.

The procedure to construct the states with particular spin-isospin symmetry is given in Ref. [19], while that for constructing wave functions with certain orbital symmetry is given in Ref. 20] for the study the Elliott model [21], with tables for the $s d, p f$ and $s d g$ shells. Finally,
TABLE IV: The number of $I=0$ states for two valence protons and two valence neutrons in the $p$ shell and the $s d$ shell with definite symmetry with respect to exchange orbital degree of freedom of two particles and the corresponding conjugate symmetry with respect to exchange spin-isospin $(S-T)$ degrees of freedom. $L$ is the total orbital angular momentum, and $S$ is the total spin. The last column gives number of the $I=0$ states with $T=0$.

\begin{tabular}{llccc}
\hline \hline$L$ & \multicolumn{1}{c}{$S$} & $I=0$ & $I=T=0$ \\
\hline \multicolumn{5}{c}{ the $p$ shell } \\
\hline$[4]$ & $0,2,4$ & 0 & 0 & 0 \\
{$[31]$} & $1,2,3$ & $0,1^{2}$ & $0^{2}$ & 0 \\
{$[22]$} & 0,2 & $0^{2}, 1,2$ & $0^{3}$ & $0^{2}$ \\
{$[211]$} & 1 & $0,1^{3}, 2$ & $0^{3}$ & 0 \\
\hline \multicolumn{5}{c}{ the $s d$ shell } \\
\hline$[4]$ & $0^{4}, 2^{5}, 3,4^{4}, 5,6^{2}, 8$ & 0 & $0^{4}$ & $0^{4}$ \\
{$[31]$} & $0^{2}, 1^{4}, 2^{7}, 3^{6}, 4^{5}, 5^{3}, 6^{2}, 7$ & $0,1^{2}$ & $0^{10}$ & $0^{4}$ \\
{$[22]$} & $0^{3}, 1,2^{5}, 3^{2}, 4^{3}, 5,6$ & $0^{2}, 1,2$ & $0^{12}$ & $0^{8}$ \\
{$[211]$} & $1^{5}, 2^{3}, 3^{5}, 4^{2}, 5^{2}$ & $0,1^{3}, 2$ & $0^{18}$ & $0^{5}$ \\
{$[1111]$} & $1,2,3$ & $0,1,2$ & $0^{2}$ & - \\
\hline \hline
\end{tabular}

the spin-isospin functions should be coupled to the orbital functions with their conjugate symmetry to obtain the fully anti-symmetric wave functions with respect to exchange two particles. The angular momentum for each state is given by coupling $S$ and $L$.

Table IV presents the number of $I=0$ states for two protons and two neutrons in the $p$ shell and the $s d$ shell with all possible symmetries with respect to orbital degree of freedom. From Table II one obtains the geometric $P_{M}$ for the $I=T=0$ states: the geometric $P_{M}$ is $-\frac{6}{5}$ for the $p$ shell and $-\frac{22}{21}$ for the $s d$ shell.

Using a TBRE Hamiltonian we obtain the following probabilities for spin- $I$ ground states: For 1000 runs, one obtains 485 and 365 runs with $(I, T)=(0,0)$ ground states for ${ }^{8} \mathrm{Be}$ and ${ }^{20} \mathrm{Ne}$, respectively. This is consistent with the result [1, 10] of the $I=T=0$ g.s. dominance in the presence of random interactions. The average value of $P_{M}$ for the $(I, T)=(0,0)$ g.s. that we obtain is -1.26 (the geometric value is $-\frac{6}{5}=-1.20$ ) and -1.66 (the geometric value is $-\frac{22}{21}=-1.05$ ) for the $p$ shell and the $s d$ shell, respectively. The average value of $P_{M}$ for a TBRE Hamiltonian and the corresponding geometric value are very close for the $p$ shell, indicating that $\alpha$-clustering correlation is not favored by random interactions. For the case of the $s d$ shell, the average value of $P_{M}$ for a TBRE Hamiltonian deviates sizably from its geometric value.

To check whether this deviation becomes larger for larger shells, we calculate the case of two protons and two neutrons in the $s d g$ shell, for which we obtained 385 cases with $(I, T)=(0,0)$ ground states among 1000 sets of the TBRE Hamiltonians. The average $P_{M}$ values for these states is -0.629 while that by assuming a random orbital symmetry is $-\frac{2}{5}$, which is close.

It is also interesting to study the distribution of overlaps between the $I=T=0$ ground state obtained from 


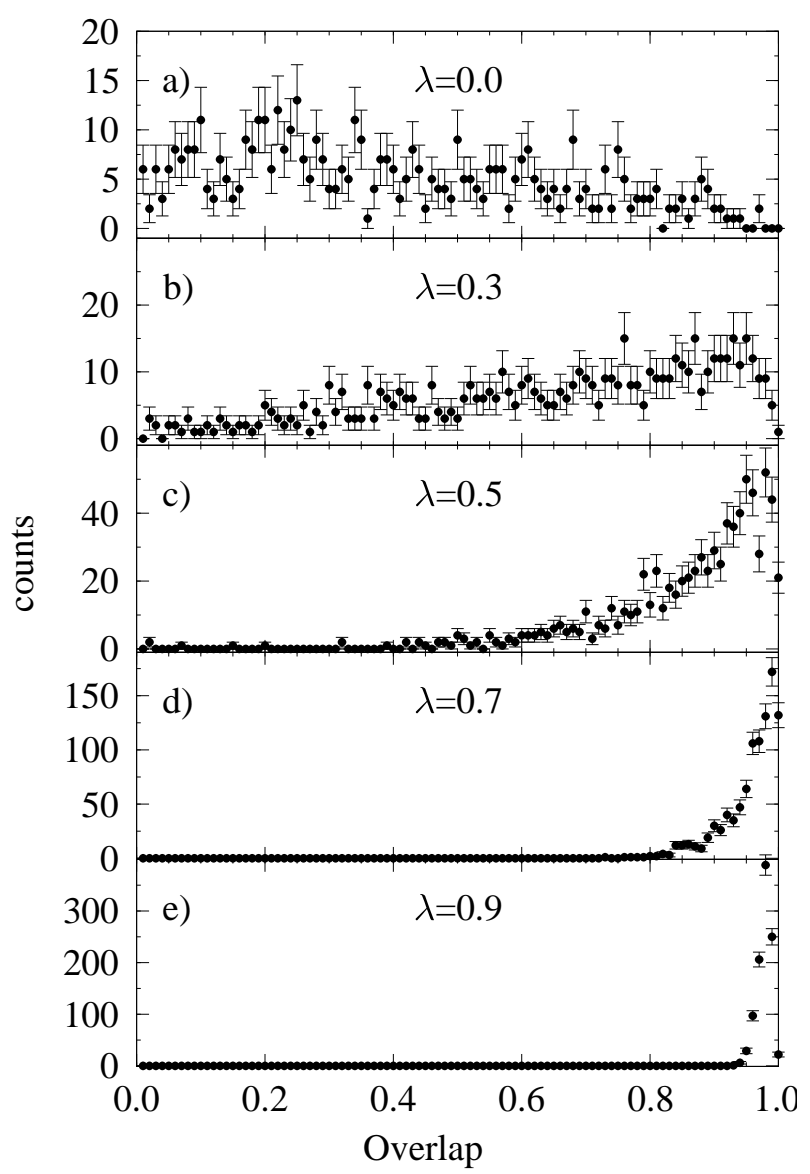

FIG. 3: The overlaps between the $I=T=0$ ground states for two protons and two neutrons in the $p$-shell obtained by Cohen-Kurath interactions and those obtained by the Hamiltonian Eq. (6). (a)-(e) corresponds to $\lambda=0,0.3,0.5,0.7,0.9$, respectively.

the realistic interactions and those obtained by pure random interactions or by a combination of realistic and random interactions. As an example we discuss here the case of two protons and two neutrons in the $p$-shell where the realistic interaction is chosen as the Cohen-Kurath interaction. We thus define a Hamiltonian

$$
H=(1-\lambda) H_{\mathrm{TBRE}}+\lambda H_{\text {real }} .
$$

Here $\lambda=0$ corresponds to the pure TBRE Hamiltonian and $\lambda=1$ corresponds to the realistic Cohen-Kurath interaction. We will vary $\lambda$ in the range from 0 to 1 , corresponding to the situation of nuclear forces with different mixtures of random noise.

The results for $\lambda=0,0.3,0.5,0.7$ and 0.9 are shown in Fig. (3) (a)-(e). The error bars indicate the statistical error in determining the numbers, defined by the square root of the number of counts for each bin. For case (a) with $\lambda=0$ one sees that the overlaps distributes "randomly" from 0 to 1 . This suggests that pure random interactions produce "random" overlaps with respect to the realistic interactions. However, for $\lambda>0.5$, the

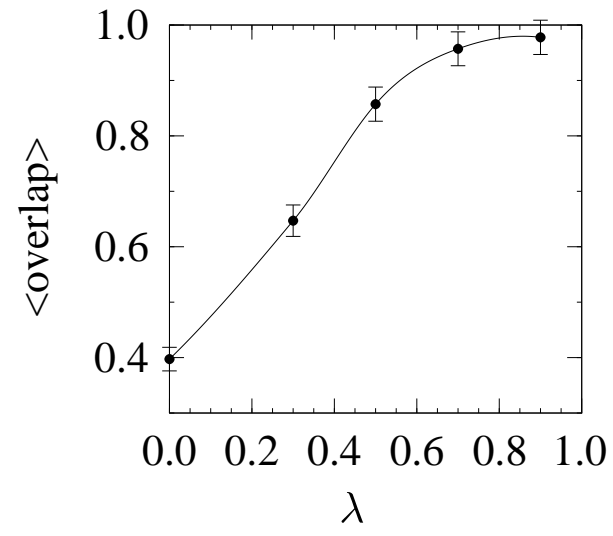

FIG. 4: Average overlap of the g.s. of the Hamiltonian of Eq. (6) with that of the realistic Hamiltonian as function of the mixing parameter $\lambda$. The line is plotted to guide the eyes.

$I=T=0$ ground states are close to that of the realistic interactions for most of the cases. This is especially clear from Fig. (4) where the overlap, averaged over the different Hamiltonians in the ensemble Eq. (6) is plotted versus $\lambda$. The statistical inaccuracies are indicated by the error bars in this figure. For values of $\lambda$ exceeding 0.6 the overlap is very close to unity while for larger admixtures of the random component in the interaction the overlap decreases approximately linearly with $\lambda$. This trend has all the signatures of a second-order phase transition. Only for limited magnitude of the random interaction the g.s. has a realistic structure which breaks down when a critical value is exceeded.

\section{DISCUSSION AND SUMMARY}

The present paper was stimulated by the discovery of the spin zero ground state dominance $(0$ g.s. $)$ of even fermion systems [1] and boson systems [2] in the presence of the random two-body ensemble (TBRE). This discovery sparked off a sudden interest of many-body systems under the TBRE. It also led to extensive studies such as energy centroid of fixed spin states, collectivity, etc. The purpose of this paper is to study the robustness of some features which are well known in nuclear physics but have not been studied under the TBRE.

First, we calculated in Sec. II the parity distribution of the g.s. for a TBRE Hamiltonian. It was found that positive parity is dominant for the g.s. of systems with even numbers of valence protons and neutrons. For odd- $A$ and doubly odd systems, the TBRE Hamiltonian leads to ground states with comparable probability for both positive and negative parity. This is similar to the global statistics for the realistic nuclei with $A \geq 70$ (refer to Table I). Unlike the spin-0 g.s. dominance in the presence of random interactions, the dominance of positive parity in the ground states of even-even nuclei has not been pointed out explicitly. Since parity is a much sim- 
pler quantity than angular momentum, an understanding of the parity dominance of even-even systems may be helpful in understanding the spin- 0 g.s. dominance of even-even nuclei in the presence of random interactions.

Second, our investigation showed that the seniority distribution for the g.s. of $s d$-shell nuclei is not dominated by low seniority components, contrary to the situation for realistic nuclei. Our investigation also suggests that the correlation between the wave function of the spin-0 g.s. for $A$ nucleons and that for $A+2$ nucleons discussed in Ref. [10] should not be understood as an indication of seniority zero component dominance.

Third, the dominance of negative external quadrupole moments at the beginning of the shell and positive quadrupole moments at the end of the shell is also observed in the g.s. obtained by using the TBRE interactions. This situation is similar to the prediction obtained from a simple harmonic oscillator potential. This means that the TBRE Hamiltonians does not lead to an overall dominance of the prolate deformation, however also in nuclei a dominance of prolate deformation is observed when both valence protons and neutrons are in the first half of a major shell.

Last, we studied the $\alpha$-clustering correlation by calculating the expectation value of the Majorana operator in the $I=0, T=0$ g.s. which are obtained using TBRE interactions. We also calculated the overlaps between the $I=0, T=0$ ground states obtained by using the TBRE Hamiltonian and the ground state by using realistic interactions. Our calculations on ${ }^{8} \mathrm{Be}$ and ${ }^{20} \mathrm{Ne}$ showed that the $\alpha$-clustering structure is not favored by a pure TBRE Hamiltonian. It is interesting to note that as function of the admixture of a realistic Hamiltonian a second-order phase transition is observed. For Hamiltonians that contain less than $\sim 0.4$ admixture of random interactions, the structure of the g.s. is close to realistic, but for higher admixtures the overlap with a realistic wave functions becomes progressively worse.

In conclusion, we have observed in this paper the dominance of positive parity in the ground states of even-even nuclei in the presence of pure random two-body interactions. Because parity is an intrinsically simpler quantum number, it will be interesting to understand the mechanism for this. In addition, it has been shown that, even though the quantum numbers for the g.s. are realistic, the dynamic properties of the ground states under the TBRE Hamiltonian, such as seniority which is a signature of pairing correlation, the $\alpha$-clustering probabilities, and the sign of quadrupole moments, are in sharp disagreement with those for realistic nuclei.

\section{Acknowledgments}

Part of this work was performed as part of the research program of the Stichting voor Fundamenteel Onderzoek der Materie (FOM) with financial support from the Nederlandse Organisatie voor Wetenschappelijk Onderzoek (NWO). This work was also supported in part by a Grant-in-Aid for Specially Promoted Research (Grant No. 13002001) from the Ministry of Education, Science and Culture in Japan. One of the authors (Y.M.Z.) acknowledges a grant from the NWO.
[1] C. W. Johnson, G. F. Bertsch, D. J. Dean, Phys. Rev. Lett. 80, 2749 (1998).

[2] R. Bijker and A. Frank, Phys. Rev. Lett. 84, 420(2000); Phys. Rev. C 62, 14303 (2000).

[3] R. Bijker, A. Frank, and S. Pittel, Phys. Rev. C 60, 021302 (1999); D. Kusnezov, N. V. Zamfir, and R. F. Casten, Phys. Rev. Lett. 85, 1396 (2000); D. Mulhall, A. Volya, and V. Zelevinsky, Phys. Rev. Lett.85, 4016 (2000); Y.M. Zhao and A. Arima, Phys. Rev. C 64, (R) 041301 (2001); R. Bijker and A. Frank, Phys. Rev. C65, 044316 (2002); P. H-T. Chau, A. Frank, N. A. Smirnova, and P. V. Isacker, Phys. Rev. C 66, (R) 061302 (2002); V. Velazquez and A. P. Zuker, Phys. Rev. Lett. 88, 072502 (2002); Y. M. Zhao, A. Arima, and N. Yoshinaga, Phys. Rev. C 66, 034302 (2002); ibid. C 66, 064323 (2002); T. Papenbrock and H. A. Weidenmueller, Phys. Rev. Lett., in press (2004); for a recent review, Y. M. Zhao, A. Arima, and N. Yoshinaga, Phys. Rep., in press (2004).

[4] D. Mulhall, A. Volya, and V. Zelevinsky, Phys. Rev. Lett. 85, 4016 (2000); Lev Kaplan, Thomas Papenbrock, and Calvin W. Johnson, Phys. Rev. C 63, 014307 (2001); M. Horoi, B. A. Brown, and V. Zelevinsky, Phys. Rev. Lett. 87, 062501 (2001); M. Horoi, A. Volya, and V. Zelevin- sky, Phys. Rev. C 66, 024319 (2002); Y. M. Zhao, S. Pittel, R. Bijker, A. Frank, and A. Arima, Phys. Rev. C 66, (R) 041301 (2002); Y. M. Zhao, A. Arima, and N. Yoshinaga, Phys. Rev. C 66, 064323 (2002); Y. M. Zhao, A. Arima, J. N. Ginocchio, and N. Yoshinaga, Phys. Rev. C 68, 044320 (2003); V. Velazquez, J. G. Hirsch, A. Frank, and A. P. Zuker, Phys. Rev. C 67, 034311 (2003).

[5] Y. M. Zhao, A. Arima, and N. Yoshinaga, Phys. Rev. C 66, 064322 (2002).

[6] R. B. Firestone, V. S. Shirley, C. M. Baglin, S. Y. F. $\mathrm{Chu}$, and J. Zipkin (eds), Table of isotopes, John Wiley and Sons, Inc (1996).

[7] Y. M. Zhao, A. Arima, and N. Yoshinaga, Phys. Rev. C68, 014322 (2003).

[8] G. Racah, Phys. Rev. 63, 367(1943); B. H. Flowers, Proc. Roy. Soc. (London) A212, 248(1952).

[9] I. Talmi, Nucl. Phys. A 172, 1(1971).

[10] C. W. Johnson, G. F. Bertsch, D. J. Dean, and I. Talmi, Phys. Rev. C 61, 014311 (1999).

[11] A. Arima, H. Horiuchi, K. Kubodera, and N. Takigawa, Advances in Nuclear Physics, Vol.5, p. 345 (Plenum press, New York, 1972).

[12] Y. Kanada-En'yo and H. Horiuchi, Prog. Theor. Phys. Suppl. 142, 205 (2001). 
[13] P. Schuck, H. Horiuchi, G. Ropke, and A. Tohsaki, nucl-th/0302076

[14] N. Tajima and N. Suzuki, Phys. Rev. C 64, 037301 (2001).

[15] R. Bijker and A. Frank, Phys. Rev. C 64, 061303 (2001);

[16] N. Auerbach, Phys. Rev. 163, 1203 (1967); there are other definitions possible for seniority number for many- $j$ shells but the essential physics remains the same.

[17] A. Bohr and B. R. Mottelson, Nuclear Structure Vol II (Benjamin, New York, 1975), p. 45; p. 136.
[18] T. Inoue, T. Sebe, H. Higawara, and A. Arima, Nucl. Phys. 59, 1 (1964).

[19] M. Hamermesh, Group Theory and its Application to Physical Problems, p. 435 (Addison-Wesley Publishing Company, INC, 1964).

[20] M. Harvey, Advances in Nuclear Physics, Vol. 1, p. 67 (Plenum Press, New York, 1968).

[21] J. P. Elliott, Proc. Roy. Soc. A 245, 128 (1958); 562 (1958). 


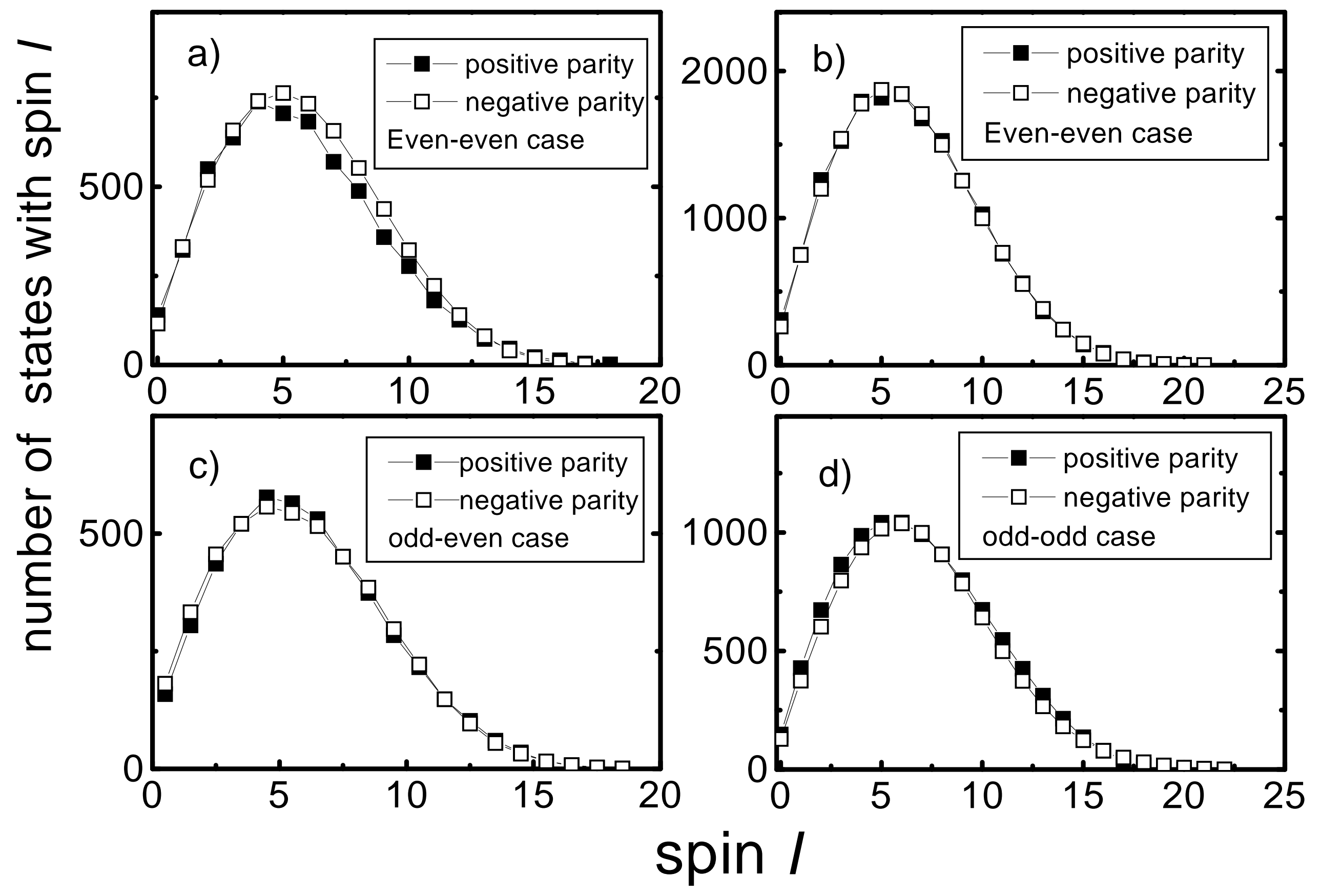

